\title{
First-List Recall as a Function of Second-List Learning Method ${ }^{1}$
}

\author{
JUDITH GOGGIN \\ Human Performance Center, University of Michigan, \\ Ann Arbor, Michigan 48104
}

\begin{abstract}
The influence of List-2 learning method on List-1 recall was studied. Lists were composed of 10 pairs of CVC stimuli and adjective responses. List I was learned by a study-test prompting method; List 2 was learned for 3 or 15 trials by either a prompting or an anticipation method. There was superior List-1 recall, as measured by an MMFR test, when a prompting method was used during List 2 and with the low degree of List-2 learning. The results were interpreted in terms of the differential opportunity for intrusions during List-2 learning.
\end{abstract}

When two incompatible responses are associated with the same stimulus, the firstlist response frequently becomes unavailable during the acquisition of the secondlist response. Using classical conditioning as an analogue, interference theory has postulated that when a stimulus is presented during List-2 learning, the first-list response occurs either overtly or covertly. Because it is not reinforced, it is extinguished or "unlearned." If this formulation is correct, overt or covert intrusions are a necessary prerequisite for extinction. There has, nevertheless, been little success in demonstrating a relationship between the two. In some cases, there are few intrusions during the learning of List 2 but a great loss in availability of List 1 ; in other instances, a high intrusion rate is correlated with little unlearning of List 1.

\footnotetext{
${ }^{1}$ This investigation was supported by a postdoctoral fellowship from the National Science Foundation and by the Advanced Research Projects Agency, Department of Defense, monitored by the Air Force Office of Scientific Research, under Contract No. AF 49(638)1235 with the Human Performance Center, University of Michigan.
}

This relationship is influenced by at least two factors: the reinforcement characteristics of the situation and list differentiation. First-list responses, when mediating the learning of second-list responses, will receive positive reinforcement if they occur as intrusions. With an A-B, A-B' paradigm, for example, where related responses are associated with the same stimulus, the first-list response is believed to mediate the learning of the second-list response. As long as they are useful in learning List 2 , the List-1 responses are not subject to negative reinforcement. Thus, the interference effects are attenuated even though overt intrusions occur more frequently than in an A-B, A-C paradigm (Barnes and Underwood, 1959). If two lists are highly differentiated, there will be little competition between the two sets of responses, and as a consequence, the amount of unlearning will decrease.

Experimental manipulations of intrusion rate have generally been limited to byproducts of manipulations of stimulus and response characteristics. Changes in degree of learning of the two lists or in response similarity, for example, alter the rate of in- 
trusions and some conclusions about the role of intrusions in unlearning have been drawn on the basis of these studies. Second-list error rate has also been manipulated by means of instructions (Keppel and Rauch, 1966), but this was not found to affect first-list recall. In the present experiment, opportunity for making intrusions is manipulated directly without changing any features of the learning material itself.

As Postman (1963) has pointed out, any strategy or procedure that reduces the opportunity for already-learned responses to occur as errors and be followed by negative reinforcement will increase the likelihood that hoth response systems will be retained. The two methods of learning that were used in this study, anticipation and prompting, were chosen because they differ in the frequency with which intrusions, both overt and covert, are likely to occur. The anticipation method in which the $S$ must guess what response is paired with the stimulus optimizes the opportunity for intrusions. The prompting method (Kopstein and Roshal, 1955), on the other hand, should reduce intrusions since the $S$ is shown the correct response immediately prior to when he must supply it. Moreover, if an intrusion does occur, the likelihood of its receiving negative reinforcement differs in the two situations. During anticipation learning, if an intrusion is given, not only will the response made be incorrect but also it will generally prevent the correct response from occurring. In the prompting method of learning, however, if an incorrect response occurs implicitly, the correct response will still be made since it is provided for the $S$. In the present experiment, all groups learned the first list by a prompting method; these groups were then divided with half learning List 2 by the prompting method and half with an anticipation procedure. This change in method for the latter groups decreases the interlist similarity and tends to work against the expected differences in List-1 recall.

A second variable, trials on List 2, was also manipulated to see if it would interact with mode of learning. If there is, in fact, no opportunity for intrusions from List 1 to occur during the learning of List 2 in the prompting method, then number of trials on List 2 should have little effect on the amount of relative retroactive inhibition (RI). With the anticipation method, however, the usual inverse relationship between number of trials on List 2 and recall of List 1 should obtain.

\section{METHOD}

Lists. The lists were composed of 10 pairs. Stimuli were CVC's of from 60 to $80 \%$ association value (Glaze, 1928). There was low intralist similarity with each vowel appearing twice and no duplication of consonants. The responses, chosen from Haagen (1942), were two-syllable adjectives. Intralist and interlist similarity were minimized by selecting adjectives from unrelated categories and having no duplication of initial letters. There were two stimulus-response pairings for each list. Each list and each pairing appeared equally often as List 1 and List 2 . There were four orders of items under both anticipation and prompting methods of learning. In addition, there were four different orders in which the stimuli alone were presented during the recall trials on List 1.

Procedure. The lists were shown on a memory drum during learning. Prompting trials and recall trials were alternated during List-1 learning for all groups. Since the $S$ s always responded correctly on prompting trials, the interspersed recall trials were necessary in order to trace the course of list acquisition. During these prompting trials, each pair was first presented together for $2 \mathrm{sec}$ and the $S$ was instructed to spell the stimulus and to pronounce the response. Immediately afterward, the stimulus was presented alone for $2 \mathrm{sec}$ and $S$ was asked to say aloud the word that just appeared with it. After $S$ had gone through all 10 pairs in this way, a 4 -sec interval intervened before the recall trial. During the recall trials, the stimuli alone were presented at a 5 -sec rate. If the $S$ remembered the responses, 
he gave them. The recall trial was followed by another 4-sec interval before the next prompting trial began. This alternate prompting-recall procedure was repeated until the criterion of one perfect recall trial was reached.

There was a 5-min interlist interval during which lists were changed and second-list instructions were read. Of the four groups, two received 3 trials of second-list learning and two received 15 trials. At each level of second-list learning, one group learned List 2 by an anticipation method (A-3, A-15) and one by a prompting method (P-3, P-15). The prompting method was identical to that described for first-list learning except that there were no recall trials. The anticipation method was similar to the conventional one. The stimulus was presented alone for $2 \mathrm{sec}$, during which $S$ was to try to guess what response was paired with it. This was followed by a 2 -sec presentation of the stimulus and response together. The $S$ was requested to spell the stimulus and to pronounce the response when they appeared together. Thus, the procedures for List-2 learning differed only in the order of the items. In the A groups, the stimulus first appeared alone, followed by the stimulus and response together; in the $\mathbf{P}$ groups, the stimulus and response appeared together first and then the stimulus appeared alcne. In both cases, the $S$ read aloud the two members of the pair when they appeared together but said only the response, if possible, when the stimulus appeared by itself. The intertrial interval was 4 sec during List-2 learning.

After the appropriate number of trials on List 2 , the $S$ s were immediately given a modified free-recall test. The stimuli were written in alphabetical order on a sheet of paper and $S$ s were requested to write down all the responses from both lists beside the appropriate stimuli in whatever order came to mind. A blank space was provided at the bottom of the page and $S$ s were urged to place here any responses whose stimulus pairings were not recalled. They were subsequently asked to identify list membership. An unlimited amount of time was given for recall.

Subiects. With four groups of $16 \mathrm{Ss}$ each, a total of $64 \mathrm{Ss}$ was used. These Ss were students at the University of Michigan and were paid for their services. No $S$ had served in another experiment where these same materials had been used. One $S$ in Group P-3 was discarded for failure to follow instructions.

The Ss were assigned to groups in blocks of four, with each block containing $1 \mathrm{~S}$ from each condition. Running order within each block was determined by a table of random numbers and, within counterbalancing restrictions, specific lists and list orders were also assigned by a table of random numbers.

\section{Results}

First-List Learning. The mean number of trials to reach the criterion of one perfect recitation on List 1 was 6.33, varying from 5.69 for Group A-15 to 7.06 for Group P-15. Although the anticipation groups tended to learn more rapidly, this difference was not significant, $F(1,60)=3.19, p>.05$. All other F's were less than 1.00 .

Transfer. There are several problems in determining the amount and direction of transfer from List 1 to List 2 in this study. The course of List- 2 acquisition for the prompting groups cannot be measured since there were no recall tests during learning. Although this same difficulty is not present for the anticipation groups, these Ss learned the two lists by different methods and the recall techniques were not comparable. Groups A-3 ( $\bar{X}=3.56$, $\mathrm{S} D=2.45)$ and $\mathrm{A}-15(\bar{X}=3.50, \mathrm{~S} D=$ 2.78) did not differ in the number of correct responses on the first three trials of List-2 learning. Beyond this, however, the data do not permit any reasonable assessment of transfer.

First-List Recall. The mean number of responses recalled for all groups on List 1 and List 2 appear in Table 1. Under stringent scoring, responses were counted correct if the response was given to the appropriate stimulus and if there was correct

TABIE 1

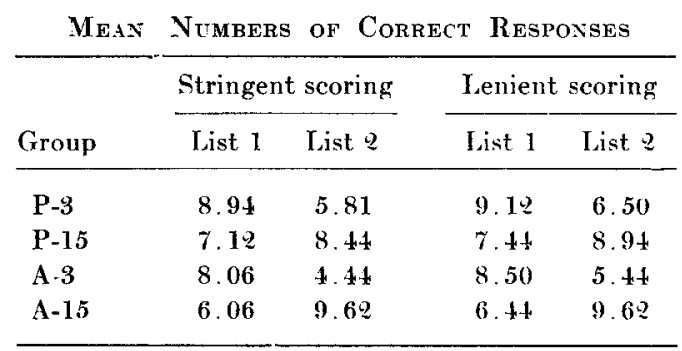


list identification. The only criterion for correctness under lenient scoring was that a response be given.

An analysis of variance on the List-1 stringent scores showed that the $\mathrm{P}$ groups recalled significantly more than the $A$ groups, $F(1,60)=4.05, p<.05$, and that recall after 3 trials on List 2 was superior to that after 15 trials, $F(1,60)=15.67$, $p<.01$. The interaction between method of learning and number of List-2 trials did not approach significance.

Second-List Recall. Results from the recall test indicated that although List-2 recall under prompting conditions was superior after 3 trials, it did not reach the same high level after 15 trials as was reached by the anticipation group. These differences were not significant, but there does seem to be a trend towards decreasing efficiency in learning with higher degrees of learning when a prompting method is used. Some experiments (e.g., Cook and Kendler, 1956) have found that prompting is generally superior to confirmation. Other studies (e.g., Hawker, 1964b; Kopstein and Roshal, 1955) demonstrate an initial superiority of the prompting method which lessens over the course of learning, but only one experiment (Hawker, 1964a) shows any tendency toward the reversal in superiority which was found here. These differences may result from the fact that in most studies of this kind tests of recall are given periodically, whereas in the present experiment no recall tests were given until the end of learning. Many Ss in Group P-15 commented on their boredom and loss of interest, and thus the inferiority of the prompting method over the longer periods of learning may be due to changes in motivation.

Error Analysis. As usual, the number of interlist intrusions made during List-2 learning was extremely small. No interlist intrusions were made by either prompting group. The A-3 group made a total of two interlist intrusions and the A-15 group made three. Although the number of interlist errors was low, the fact that none were made by the prompting groups supports the hypothesis that the tendency to make intrusions is minimized by this method of learning.

\section{Discusston}

The results of this experiment point to the importance of method of learning an interfering list as a contributor to relative RI. List- 1 recall is less when List 2 is learned by an anticipation method than when it is learned by a prompting method. The most obvious explanation of these differences is in terms of the greater opportunity to make intrusions, which would make the List-1 associations for the anticipation groups more susceptible to the unlearning process. This conclusion is supported by the fact that there were some interlist intrusions in the A groups, whereas none occurred in the $P$ groups.

There are indications that the prompting method does not completely eliminate RI. The recall of List 1 after three List- 2 trials was only 8.94 , and it is probable that recall would have been nearly perfect had there been no interpolated learning. Additional losses in recall of List 1 were demonstrated between 3 and 15 trials of List-2 learning. This suggests that some implicit intrusions may occur even during prompting learning. When questioned at the end of the experiment, some of the $S$ s in Group P-15 said that they had "put List 1 out of their mind at first but had attempted to recall the List-1 responses during the latter part of List-2 acquisition."

It has already been pointed out that one factor which may have decreased the magnitude of the difference in List-1 recall between $A$ and $P$ groups is that learning in the $\mathbf{P}$ groups was very similar for Lists 
1 and 2. Both lists were learned by a prompting method, although in the case of List 1 tests of recall were interspersed between the learning trials. The method of learning Lists 1 and 2 for the A groups differed. List 1 was learned by a prompting method and List 2 was learned by an anticipation method. This change in learning method should have reduced the generalized response competition (Postman, 1961) for the A groups and, as a result, helped to counteract the detrimental effect of anticipation learning. A design that varied both first-list and second-list learning method could be used to evaluate the effect of this procedure change. Since the predicted differences were found in the present study, one would expect even larger differences when method change was controlled.

\section{REFERENCES}

Barnes, J. M., AND Underwood, B. J. "Fate" of first-list associations in transfer theory. $J$. exp. Psychol., 1959, 58, 97-105.

Cook, J. O., ANd Kendler, T. S. A theoretical model to explain some paired-associate learning data. In G. Finch and F. Cameron (Eds.), Symposium on Air Force human engineering, personnel and training research. Washington, D.C.: Nat. Acad. Sci.-Nat. Res. Coun., Pub. 455, 1956. Pp. 90-98.

GLAzE, J. A. The association value of nonsense syllables. J. genet. Psychol., 1928, 35, 255269.

HAagen, C. H. Synonymity, vividness, familiarity, and association-value ratings for $\mathbf{4 0 0}$ pairs of common adjectives. J. Psychol., 1949, 30, $185-200$.

HAwkER, J. R. Effects of prompting and confirmation in a serial learning task. J. exp. Psychol., 1964, 67, 99-101. (a)

HAwken, J. R. The influence of training procedure and other task variables in paired-associate learning. J. verb. Learn. verb. Behav., 1964, 3, 70-76. (b)

Keppel, G., and Rauch, D. S. Unlearning as a function of second-list error rate. $J$. verb. Learn. verb. Behav., 1966, 5, 50-58.

Kopstein, F. F., and Roshal, S. M. Method of presenting word pairs as a factor in foreign vocabulary learning. Amer. Psychologist, 1955, 10, 354. (Abstract)

Postman, L. The present status of interference theory. In C. N. Cofer (Ed.), Verbal learning and verbal behavior. New York: McGrawHill, 1961.

Postman, L. Does interference theory predict too much forgetting? J. verb. Learn. verb. Behav., 1963, 2, 40-48.

(Received August 25, 1965) 